\title{
ON THE INFLUENCE OF TRANSMISSION LINE ON COMMUNICATION SYSTEM USING CHAOS SYNCHRONIZATION
}

\author{
Junji KAWATA ${ }^{1} \quad$ Yoshifumi NISHIO ${ }^{2}$ Akio USHIDA ${ }^{3}$ \\ ${ }^{1}$ Dept. of Information Science and Systems Eng., Tokushima Bunri University \\ kawata@me.bunri-u.ac.jp \\ ${ }^{2,3}$ Dept. of Electrical and Electronic Eng., Tokushima University \\ nishio@ec.tokushima-u.ac.jp, ushida@ee.tokushima-u.ac.jp
}

\begin{abstract}
In this study, a transmitter-receiver system utilizing chaos synchronization is investigated, where transmitter and receiver subsystems are coupled by a transmission line, assuming practical implementation. The influence of both loss of transmission line and mismatching on synchronization is examined by computer simulation using the method of characteristics.
\end{abstract}

\section{INTRODUCTION}

Recently chaos synchronization has received a great deal of attention since its discovery by Pecora and Carroll [1]. A number of recent papers have been published in the area and many secure communication techniques [2]-[4] also have been dcveloped, which may be useful for practical application. However the transmitter and receiver are only directly coupled through voltage buffer in [2]-[3]. For practical implementation of these secure communication techniques, the subsysterns need to be interconnected by a transmission line. On the other hand, a digital secure communication system via DSP implementation [4] has been developed, where the transmitter and receiver are interconnected by a digital transmission line. The authors [4] have also discussed the disadvantage with the use of analog transmission linc: the influence of noise and gain distortion. It seems very important that the effect of transmission line has on communication system is investigated, for practical implementation.

Accordingly, in this article, we investigate the influence of the loss of an analog transmission line and mismatching on synchronization in a transmitter-receiver system based on the concept of Pecora and Carroll. The system is shown in Fig. 1. As widely used in [2]-[3], we also use Chua's circuit as the transmitter and receiver, which are coupled by an analog transmission line with matching impedance. We analyze phenomena observed in the system numerically, because it includes a transmission line. For the purpose, we use the method of characteristics [5] which has been proposed for the transient simulation of lossless transmission lines. In the method of characteristics a lossless transmission line is modeled as the characteristic model. For lossy transmission lines, a brute-force approach [6] is used, where the lossy transmission line is modeled by several cascaded cells. Fach cell consists of lumped resistors and a lossless line which is replaced by its characteristic model. Thus the transient response can be calculated easily by computer simulation with the numerical integration method such as Runge-Kutta method.

We first show by computer calculation that the subsystems synchronize when the transmission line is lossless. Then the influence of loss on chaos synchronization is investigated for lossy transmission line. Further the influence of matching error on that is also examined. It is shown by numerical experiments that synchronization breaks more frequently than we had expected, as loss increases.

\section{CIRCUIT MODEL AND}

Fig. 1 shows a transmitter-receiver system based on the Pecora-Carroll concept. Each subsystem is based on Chua's circuit whose chaotic behavior has been widely studied (see references in [8]). The transmitter and receiver are interconnected by a transmission line, where a matching impedance is connected at the receiving side to avoid signal distortion due to reflection.

Chaotic signal $v_{C 1}$ is transmitted to the receiver through the transmission line. After some time delay $\tau$, the transmitted signal reaches at the receiving end. The voltage $\hat{v}_{C 1}(t)$ at the end will be equal to $v_{C 1}(t-\tau)$ if the transmission line is lossless and its characteristic impedance is completely matched to the matching impedance. The first (second) receiver subsystem then reproduce $v_{C 2}^{\prime}(t)\left(v_{C 1}^{\prime}(t)\right)$ which will be a copy of $v_{C 2}(t-\tau)\left(v_{C 1}(t-\tau)\right)$, only when components of the subsystem are exactly identical to the transmitter (first receiver) subsystem.

As this system includes the transmission line, we can not investigate the system analytically. Therefore the system will be just analyze numerically using the method of characteristics. A lossless transmission line can be modeled using the charactcristic model [5]. Replacing the lossless transmission line by the characteristic model, the equivalent circuit as shown in Fig. 2 can be obtained, which yields the following system equations:

$$
\begin{aligned}
C_{1} \frac{d v_{C 1}}{d t} & =G\left(v_{C 2}-v_{C 1}\right)-g\left(v_{C 1}\right), \\
C_{2} \frac{d v_{C 2}}{d t} & =G\left(v_{C 1}-v_{C 2}\right)+i_{L}, \\
L \frac{d i_{L}}{d t} & =-v_{C 2}, \\
\hat{v}_{C 1} & =e_{2}(t-\tau) \cdot Z_{m} /\left(Z_{0}+Z_{m}\right), \\
e_{1}(t) & =2 \hat{v}_{C 1}-e_{2}(t-\tau), \\
e_{2}(t) & =2 v_{C 1}-e_{1}(t-\tau), \\
C_{2} \frac{d v_{C 2}^{\prime}}{d t} & =G\left(\hat{v}_{C 1}-v_{C 2}^{\prime}\right)+i_{L}^{\prime}, \\
L \frac{d i_{L}^{\prime}}{d t} & =-v_{C 2}^{\prime}, \\
C_{1} \frac{d v_{C 1}^{\prime}}{d t} & =G\left(v_{C 2}^{\prime}-v_{C 1}^{\prime}\right)-g\left(v_{C 1}^{\prime}\right),
\end{aligned}
$$

where $g(\cdot)$ is a piecewise linear function defined by:

$$
g(v)=m_{1} v+\frac{1}{2}\left(m_{0}-m_{1}\right)\left[\left|v+B_{p}\right|-\left|v-B_{p}\right|\right] .
$$

Eqs. (1)-(3), (7)-(8) and (9) describe the dynamics of the transmitter, the first receiver, and the second receiver subsystem, respectively, while Eqs. (4)-(6) are derived from the characteristic model [5]. 
For the case of lossy transmission line, Eqs. (4)-(6) must be replaced by the corresponding state equations, because the lossy transmission line is approximately modeled by several cascaded cells. Each cell consists of lumped resistors and a lossless line which is further replaced with its characteristic model (see [6] for detail). In [7] a theoretic justification for this approach as well as an error analysis in the frequency domain have been reported. Based on the error analysis we can decide the number of cells we need for a prescribed modeling error.

To investigate the synchronization in the transmitterreceiver system, let us fix the parameters of the transmitter so that it exhibits a chaotic attractor. From Chua's circuit, the double scroll attractor can be observed for the following parameters:

$$
\begin{aligned}
& R=1 / G=1700[\Omega], \quad C_{1}=10[\mathrm{nF}], \quad C_{2}=100[\mathrm{nF}], \\
& L=18[\mathrm{mH}], \quad m_{0}=-0.75[\mathrm{mS}], \quad m_{1}=-0.41[\mathrm{mS}], \\
& B_{p}=1[\mathrm{~V}] .
\end{aligned}
$$

These values are fixed throughout the following discussion.

We suppose here that the parameters of both transmitter and receiver subsystems are completely identical. First, let's examine the system for the case that the transmission line is lossless and has the following parameters.

$$
Z_{0}=50[\Omega], \quad \tau=0.5[\mathrm{msec}]
$$

Using the method of characteristics [5], the computer simulation is carried out, where we use 4 th order Runge-Kutta method (stepsize is 0.00001 [msec]) throughout the following simulations. Fig. 3 shows the phase portrait. In the figure, it should be noted that the horizontal axis in (b) and the vertical axis in (c) are the time-delayed voltage $v_{C 1}^{\prime}$, and the vertical axis in (b) is the time-delayed voltage $v_{C_{2}}^{\prime}$. As we had expected, the transmitted signal $v_{C_{1}}$ and the reproduced signal $v_{C 1}^{\prime}$ synchronized completely. For arbitrary values of $Z_{0}, \tau$ different from above example, they also synchronized completely.

\section{INFLUENCE OF LOSS}

Since a transmission line such as a coaxial cable has loss normally, we need to investigate the system for the case of lossy transmission line. In this case the time-delayed signal $\hat{v}_{\mathrm{C} 1}$ at the receiving end is different from the transmitted signal $v_{C 1}$ because of attenuation. In the simulation we used the following set of parameters.

$R_{d}=20[\Omega / \mathrm{km}], \quad L_{d}=0.25[\mathrm{mH} / \mathrm{km}], \quad C_{d}=100[\mathrm{nF} / \mathrm{km}]$, $G_{d}=0[\mathrm{~S} / \mathrm{km}]$

The per-unit-length parameters of a real coaxial cable is probably the almost same as above one. Based on the error analysis [7] we evaluated the modeling error and then decided $N$ so that it is within $0.1 \%$ in a prescribed frequency range. We calculated the value of matching impedance by the following equation:

$$
Z_{m}=\left|Z_{0}(j \omega)\right|=\left|\sqrt{\frac{R_{d}+j \omega L_{d}}{G_{d}+j \omega C_{d}}}\right|,
$$

where the value of $\omega$ is supposed to be the natural angular frequency of Chua's circuit decided by $L$ and $C_{2}$. See Fig. 4 . Roughly speaking, the subsystems synchronize yet. Further we examine the system for larger loss. To investigate the effect of loss, we fix the per-unit-length parameters and then simulate the system by varying the line length. In this case the total loss of transmission line increases with the length. For larger loss, the subsystems did not synchronize unfortunately, and obvious differences in the phase plain were not found. In order to investigate the effect of increasing loss fully, thus, we examined the time variation of difference between the voltages $v_{C 1}$ and $v_{C 1}^{\prime}$ for various length. Some results are shown in Fig. 5. As we had expected, it is clear that synchronization breaks more frequently as loss increases.

\section{INFLUENCE OF MISMATCHING}

Here we will investigate the effect of mismatching has on synchronization, because it may be very difficult that $Z_{m}$ is completely matched to $Z_{0}$ in real circuit. Adding an error factor to the matching impedance, the influence of matching error is examined. In this case the reflection due to mismatching takes place at the receiving end and consequently the transmitted signal is affected by the distortion. The difference of voltages $v_{C 1}$ and $v_{C 1}^{\prime}$ is plotted in Fig. 6 for $5 \%$ error. For lossless transmission line, synchronization largely breaks at times, while remarkable difference by matching error is not observed for lossy transmission line (Compare Fig. 5(a),(b) with Fig. 6(b),(c)). This reason will be that the reflection waveforms induced by mismatching is also attenuated because of loss.

\section{CONCLUSION}

In this article we have investigated the influence of transmission line on a transmitter-receiver system using chaos synchronization. It is found by numerical experiments that the transmitter and receiver synchronize roughly for relatively smaller loss and that desynchronization occurs more frequently as loss increases. It also seems that for lossy transmission line synchronization suffers from loss than mismatching due to attenuation.

We will investigate whether the subsystems synchronizo independently of loss, when the attenuated signal $\hat{v}_{C 1}$ is properly amplified using an amplifier at the receiver subsystem. We also intend to study how demodulated signal is affected by both loss and mismatching, when a information signal is added to our system and then modulated by chaotic signal.

\section{REFERENCES}

[1] L. M. Pecora and T. L. Carroll, "Synchronization in chaotic systems," Phys. Rev. Lett., vol. 64, no. 4, pp. 821-824, 1990.

[2] L. Kocarev et al., "Experimental demonstration of secure communication via chaotic synchronization," Int. J. Bifurcation Chaos, vol. 2, pp. $709 \cdot 713,1992$.

[3] H. Dedieu, M. P. Kennedy and M. Hasler, "Chaus shift keying: Modulation and demodulation of a chaotic character using self-synchronizing Chua's circuits," IEEE Trans. Circuits Syst. II, vol. 40, no. 10, pp. 634-642, Oct. 1993.

[4] H. Kamata, T. Endo and Y. Ishida, "Secure communication system using chaos via DSP implementation," Proc. IEEE ISCAS '96, vol. 3, pp. 112-115, 1996.

[כ] F. H. Branin, Jr., "Transient analysis of lossless transmission line," Proc. IEEE, vol. 55, pp. 2012-2013, Nov. 1967

[6] H. W. Dommel, "Digital computer solution of electromagnetic transients in a single or multiphase networks," IEEE Trans. Power App. Syst., vol. PAS-88, pp. 388-399, Apr. 1969

[7] R. Gupta and L. T. Pileggi, "Modeling lossy transmission lines using the method of characteristics," IEEE' Trans. Circuits Syst., vol. 43, no. 7, pp. 580-582, July 1996.

[8] L. O. Chua, "Chua's circuit: Ten years later," IEICE Trans. Fundamentals, vol. E77-A, no. 11, pp. 1811 1821, Nov. 1994 


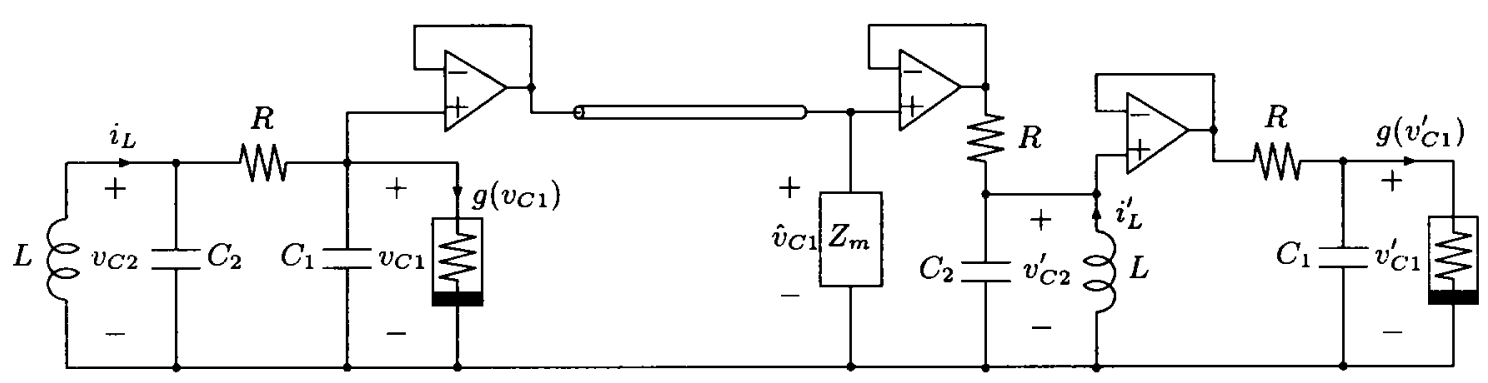

Transmitter subsystem

First receiver subsystem

Second receiver subsystem

Figure 1. Transmitter-receiver system using Chua's circuit.

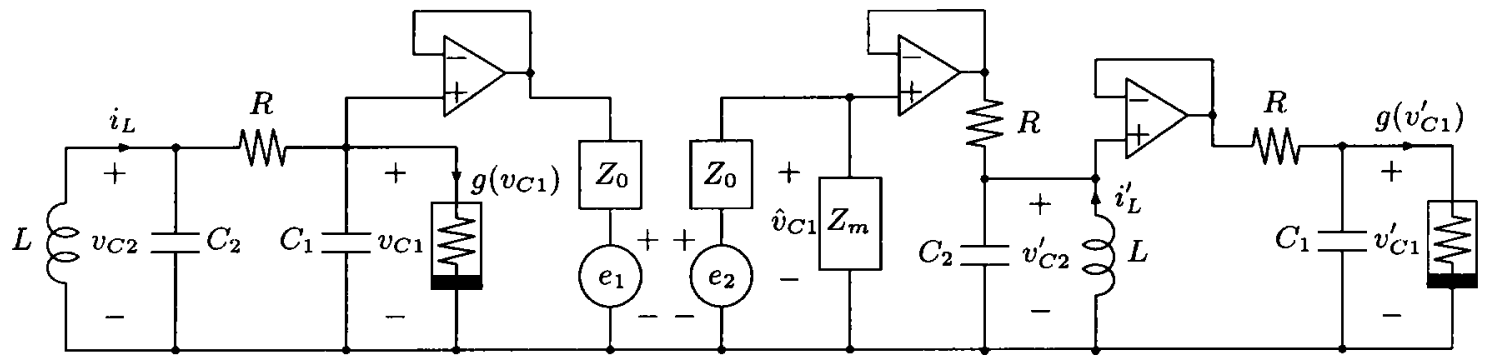

Figure 2. Equivalent circuit for the system shown in Fig. 1.

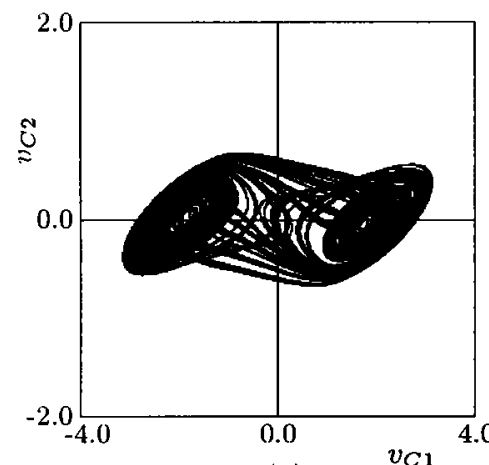

(a)

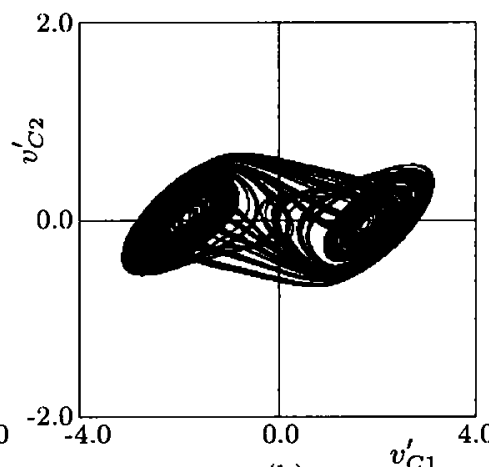

(b)

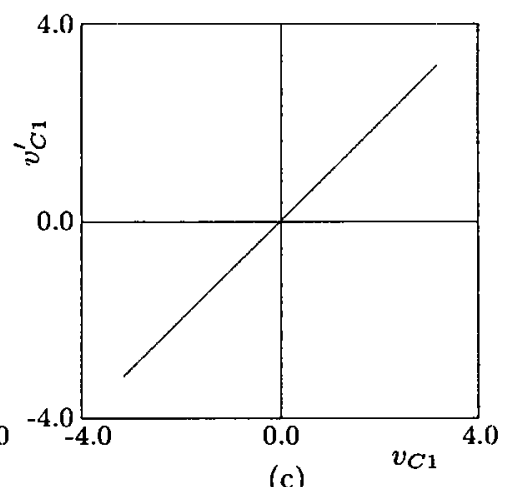

(c)

Figure 3. Synchronization of the system for the case that the transmission line is lossless: Phase portrait in (a) $v_{C 1}-v_{C 2}$ plane, (b) $v_{C 1}^{\prime}-v_{C 2}^{\prime}$ plane, (c) $v_{C 1}-v_{C 1}^{\prime}$ plane, respectively.

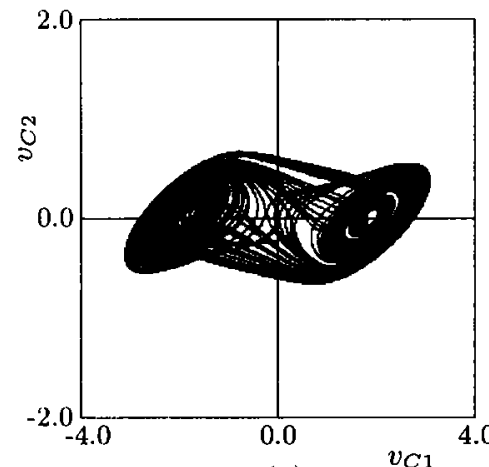

(a)

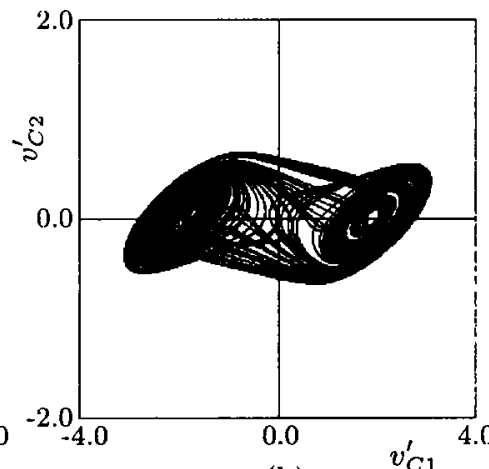

(b)

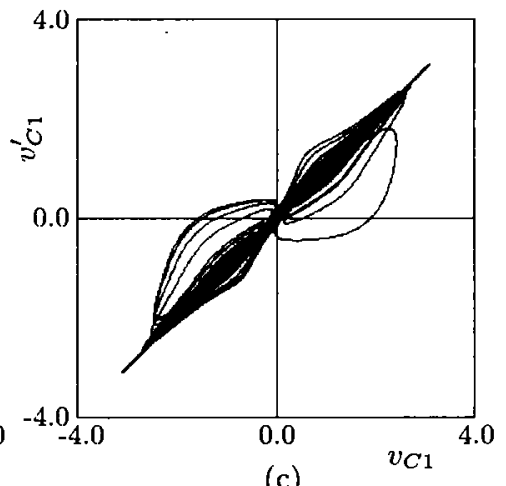

(c)

Figure 4. Synchronization of the system for $0.01[\mathrm{~km}]$ lossy transmission line: Phase portrait in (a) $v_{C 1}-v_{C 2}$ plane, (b) $v_{C 1}^{\prime}-v_{C 2}^{\prime}$ plane, (c) $v_{C 1}-v_{C 1}^{\prime}$ plane, respectively. 

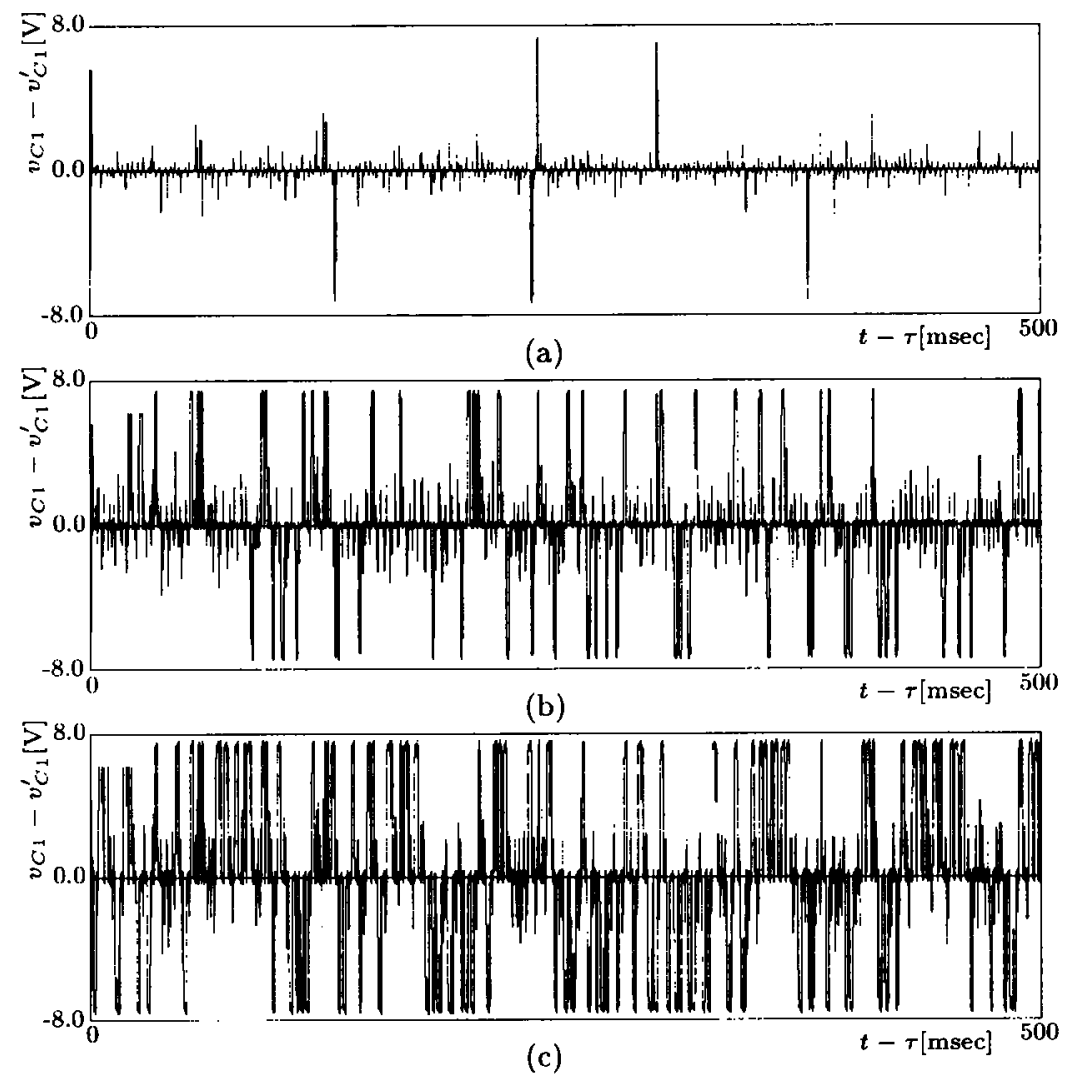

Figure 5. Time evolution of $v_{C 1}-v_{C 1}^{\prime}$ with the variation of length (loss). (a) $0.1[\mathrm{~km}]$, (b) $0.5[\mathrm{~km}]$, (c) $1[\mathrm{~km}]$.
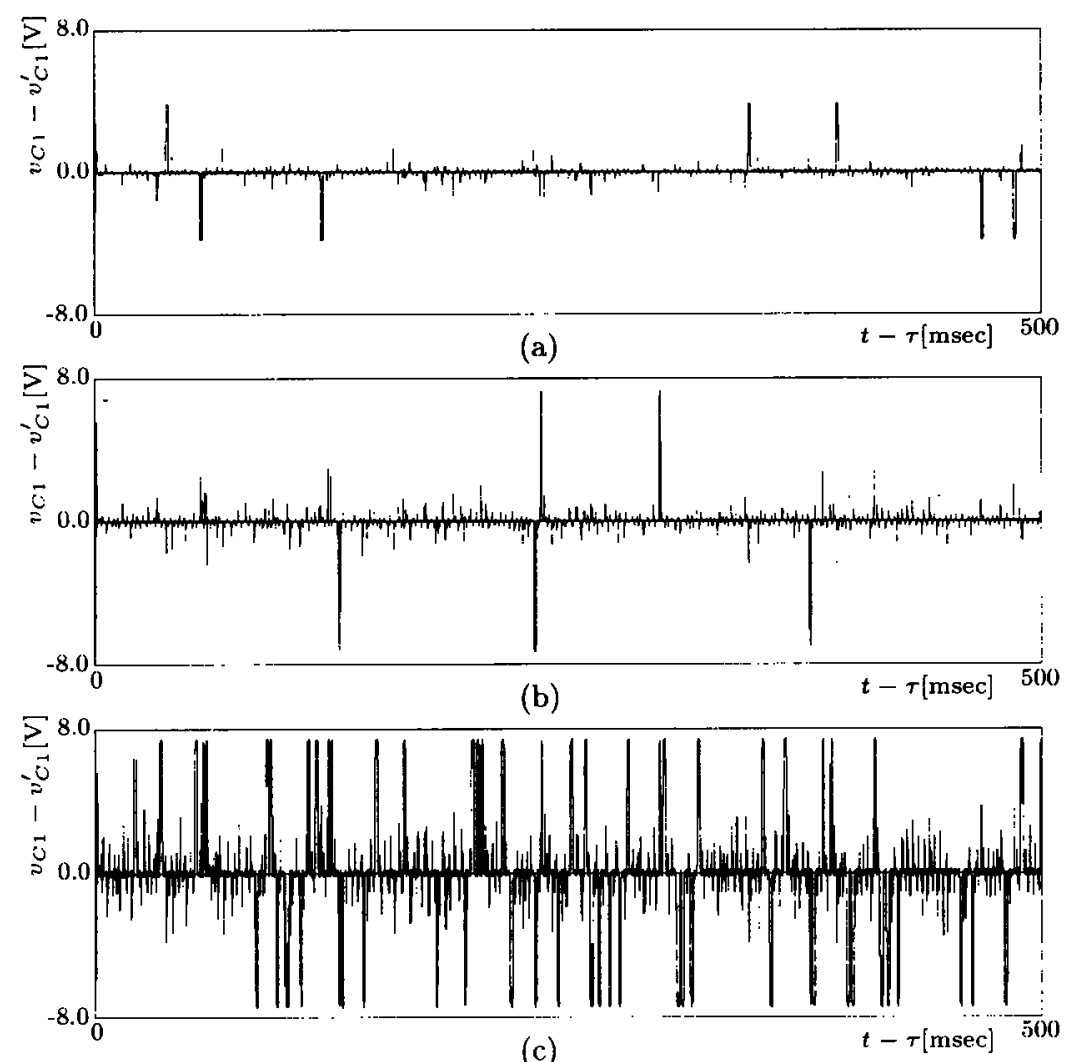

Figure 6. The influence of matching error $5 \%$ for (a) lossless line, and lossy line with length (b) $0.1[\mathrm{~km}]$, (c) $0.5[\mathrm{~km}]$. 